\title{
Broken symmetry phase transition in solid HD: a manifestation of quantum orientational melting
}

\author{
Yu. A. Freiman and S. M. Tretyak \\ B. Verkin Institute for Low Temperature Physics and Engineering, 47 Lenin Ave., 310164 Kharkov, Ukraine \\ E-mail: freiman@ilt. kharkov.ua
}

\section{A. Jeżowski}

Trzebiatowski Institute for Low Temperatures and Structure Research, Polish Acad. of Sci., P.O. Box 937, 50-950 Wroclaw 2, Poland

E-mail: an_je@int.pan.wroc.pl

R. J. Hemley

Geophysical Laboratory and Center for High Pressure Research, Carnegie Institution of Washington, 5251 Broad Branch Road NW Washington, DC 20015, USA

E-mail: hemley@gl.ciw.edu

Received March 19, 1998

\begin{abstract}
Theoretical study of the broken symmetry phase (BSP) transition line in solid HD reveals that its anomalous features provide evidence for quantum orientational melting. The observations of unusual reentrant behavior is a consequence of the symmetry properties of the system, namely, the fact that in HD all rotational states and transitions between them are allowed, in contrast to the behavior of the homonuclear isotopes $\mathrm{H}_{2}$ and $\mathrm{D}_{2}$. The systematic underestimation of the transition pressure characteristic of all theories of the BSP transition can be removed if crystal-field effects are taken into account.
\end{abstract}

PACS: 67.80.-s, 62.50.+p, $31.70 . \mathrm{Ks}$

\section{Introduction}

The large isotopic family of hydrogens $\left(\mathrm{H}_{2}\right.$, $\mathrm{HD}, \mathrm{D}_{2}, \mathrm{HT}, \mathrm{DT}, \mathrm{T}_{2}$ ) presents a unique possibility for studying the diversity of quantum isotopic effects [1]. The differences in properties of the isotopic substances cannot be, as a rule, related solely to the de Boer quantum parameter, and symmetryrelated nuclear-spin effects turn out to be far more essential. Due to symmetry requirements, hydrogen and deuterium have two species: para- $\mathrm{H}_{2}$ and ortho$\mathrm{D}_{2}$ correspond to the even rotational quantum number $J$, whereas ortho- $\mathrm{H}_{2}$ and para- $\mathrm{D}_{2}$ correspond to odd $J$ states. In the case of $\mathrm{HD}$ molecules, the nuclei are distinguishable and the molecules do not possess a center of inversion. As a result, the HD molecules do not have ortho-para species and all angular momentum states $J=0,1,2, \ldots$ and transitions between all of them are allowed.

To a very good approximation, the electron density distributions in the $\mathrm{H}_{2}$ and $\mathrm{HD}$ molecules are the same. But in the HD molecule the center-ofcharge does not coincide with the center-of-mass. Since the molecule rotates around its center-of-mass but the intermolecular interactions are related to the center-of-charge, rotations of the molecules are accompanied by translational displacements of the center-of-mass. Thus, the rotation and translation of the molecule are coupled dynamically. As a result of such off-center rotation, an additional Heisenberglike term appears in the anisotropic part of the intermolecular potential, as first has been shown by van Kranendonk [2]. Evidence for differences in properties of asymmetric and symmetric hydrogens, which are a consequence of the coupling of the rotation and translation of the molecule in the condensed state, has been reported since the sixties [3]. A large negative deviation of the $c / a$ ratio from the ideal hep value of $\sqrt{8 / 3}$ found by Prokhvatilov et al. [4] for HD at zero pressure by x-ray diffraction was attributed by the authors to features of the intermolecular interaction of HD molecules. 
Subsequent calculations by Strzhemechny [5] support this conclusion.

But the most striking differences between the homonuclear hydrogens $\left(\mathrm{H}_{2}\right.$ and $\left.\mathrm{D}_{2}\right)$ and $\mathrm{HD}$ are evident by properties of the solids under very high pressures. At low pressure, the free rotor quantum numbers $J M$ remain good quantum numbers for molecules in solid $\mathrm{H}_{2}$ and $\mathrm{D}_{2}$, and at low temperatures only lowest states $J=0$ in the even $J$ species are occupied. Since the $J=0$ state has a spherically symmetric spatial distribution, there is no orientational order in $p-\mathrm{H}_{2}$ and $o-\mathrm{D}_{2}$ at low pressures down to $T=0 \mathrm{~K}$. The intermolecular interaction admixes the higher rotational states into the ground state wave function, but this admixture is too small to produce the ordering at zero pressure. With increasing pressure, the anisotropic intermolecular interaction increases, and admixtures of higher rotational states into the ground state wave function become more appreciable, eventually resulting in the transition into a phase characterized by orientational order. This transition has been called the broken symmetry phase (BSP) transition. This transition was foretold by Raich and Etters in 1972 [6] and found experimentally by Silvera and Wijngaarden in 1981 in $o-\mathrm{D}_{2}$ [7] and then by Lorenzana, Silvera and Goettel in 1989 in $p-\mathrm{H}_{2}$ [8]. Moshary, Chen and Silvera [9] experimentally studied the BSP transition in HD and reported evidence for a nonmonotonic phase line (i.e., a $P-T$ minimum and thus reentrant behavior) that contrasted markedly with that found for $p-\mathrm{H}_{2}$ and $o-\mathrm{D}_{2}$.

The BSP transition in HD was found at 68.3 $\mathrm{GPa}$ and $3 \mathrm{~K}$, which gave by extrapolating the temperature dependence a transition pressure $P_{\mathrm{tr}}$ at $T=0 \mathrm{~K}$ of $P_{0} \approx 69.0( \pm 2) \mathrm{GPa}$. The minimum point was located at $P_{m} \approx 53 \mathrm{GPa}$ and $T_{m} \approx 30 \mathrm{~K}$ or in reduced units at $T_{m} / B \approx 1 / 2$ ( $B$ is the rotational constant). The disordered phase is reentrant, that is, for fixed pressure in the range between $P_{m}$ and $P_{0}$, as temperature is increased, the solid goes from a disordered to an ordered and then to a disordered phase once again. The slope of the orientational melting curve $d P_{\mathrm{tr}} / d T$ is negative at temperatures less than $T_{m}$ and positive at $T>T_{m}$. At $T \approx 65 \mathrm{~K}$ $(T / B \approx 1)$ the transition pressure becomes equal to $P_{0}$. Above this temperature, the studied portion of the transition curve is approximately linear with temperature.

The peculiar features of HD responsible for the remarkable behavior of its BSP transition line and the nature of the transition itself are thus of obvious interest. Two different mechanisms have been proposed in the literature. One of them, called quan- tum orientational melting $[10,11]$, was studied for the model system of all- $J$ quantum rotors before the phase transition in HD was found experimentally. A different approach to the problem was proposed by Strzhemechny [5]. According to the latter model, the mechanism of the pressure-driven orientational ordering in solid HD is completely different from that in $\mathrm{H}_{2}$ and $\mathrm{D}_{2}$, and is related to the creation of a single delocalized $J=1$ state that is a direct analog of the zero-point vacancy waves in quantum crystals. Thus, this mechanism is directly tied with the Heisenberg-like term in the intermolecular potential that is specific to HD and its off-center rotation.

The BSP transition has attracted considerable interest from both experiment and theory for many years (see, for example, [1,12-14] and references therein). The main efforts in theory have been made either to calculate the transition pressure at zero temperature [15-20] or to predict the lattice structure for the BS phase [21-26]. After the BSP transition was found experimentally, it became clear that the critical densities determined in the first theoretical studies $[6,15,16]$ were considerably underestimated relative to experiment. In more recent work, several basic assumptions and simplifications of these early treatments have been subjected to careful analysis, and attempts were made to go beyond the most questionable approximations. One of the most significant approximations is the mean field (MF) approximation. The effect of correlations neglected in the MF approximation was taken into account (in different ways) by Lagendijk and Silvera [18] and by Sprik and Klein [19], the effect of translation-rotation coupling was studied by Janssen and van der Avoird [20], and the consequences of different forms of the intermolecular potential were tested by Aviram et al. [17]. Though a number of important results emerged from these studies, only small changes were found in the predicted transition pressure. It was suggested [20] that many-body effects are responsible for the systematic underestimation of the transition pressure. In the present paper, we propose a new approach to this problem. It is shown that the main discrepancy between theory and experiment can be removed even in the MF approximation if crystal field effects are taken into account.

Another problem that has been intensively studied in the context of the BSP transition is the question of the structure of the BS phase. In early theoretical studies $[6,15,17,18]$, the structure assumed to be $P a 3 \alpha$-nitrogen structure. The crystal structure of the high-temperature phase to $120 \mathrm{GPa}$ 
(phase I) was found to be hcp [21], but the structure of the BS is still unknown. Recent theoretical search for the lowest-energy structure of the BS phase based on the local density approximation [22-24] and $a b$ initio molecular dynamics simulations [25, 26] suggest a four-molecular orthorhombic structure of $P c a 2_{1}$ symmetry. On the other hand, recent spectroscopic data do not rule out $\mathrm{Pa} 3$, at least for $p-\mathrm{H}_{2}$ [27].

In the present paper the phase diagram of solid HD has been calculated. We show that quantum orientational melting can readily account for the unusual features of the BSP transition in this system. The behavior of the phase transition line in solid $\mathrm{HD}$ as compared with $\mathrm{H}_{2}$ and $\mathrm{D}_{2}$ is a consequence of the symmetry properties of the system, namely of the fact that in HD transitions between all the rotational states are allowed in contrast to $\mathrm{H}_{2}$ and $\mathrm{D}_{2}$.

\section{Basic equations}

In the MF approximation, the Hamiltonian of the system of linear rotors interacting via quasiquadrupolar forces can be written in the following form [28]:

$$
\mathcal{H}=B L^{2}-\left(U_{0} \eta+U_{1}\right) Y_{20}+U_{0} \eta^{2} / 2,
$$

where $L$ is the operator of angular momentum; $U_{0}$ and $U_{1}$ are molecular and crystal field constants; $B$ is the rotational constant; $\eta=\sqrt{4 \pi / 5}<Y_{20}>$ is the order parameter; and <. . .> denotes thermodynamic averaging with the Hamiltonian [Eq. (1)].

The MF constant

$$
U_{0}=-\sum_{f f^{\prime}} \sum_{\alpha \beta \gamma \delta} V_{f f^{\prime}}^{\alpha \beta \delta \delta} Q_{f}^{\alpha \beta} Q_{f^{\prime}}^{\gamma \delta},
$$

where $V_{f f}^{\alpha \beta \gamma \delta}$ 'is the interaction matrix, defined by the parameters of the intermolecular potential and by the lattice parameters; $Q_{f}^{\alpha \beta}=\Omega_{\alpha} \Omega_{\beta}-1 / 3 \delta_{\alpha \beta}, f$ numbers the lattice sites; $\Omega$ is a unit vector along the equilibrium orientation of the molecule in the site $f$.

While the molecular field is generated by the coupling terms in the intermolecular interaction potential, the crystal-field term [2]

$$
U_{1}=-\frac{4 \pi}{5} \sum_{\delta} B\left(R_{\delta}\right) Y_{20}\left(\Omega_{\delta}\right)
$$

originates from single-molecular terms in the intermolecular potential. Here $B\left(R_{\delta}\right)$ is the radial function characterizing the anisotropic pair potential [2]; $\mathbf{R}_{\delta}$ is the radius-vector of the nearest neighbors, $\Omega_{\delta}=\mathbf{R}_{\delta} / R_{\delta}$, and $\delta$ are indices of the nearest neighbors.

The orientational state of the system is determined by values and signs of the molecular and crystal constants, and can be described by positive and negative order parameters. In the case of $\alpha-\mathrm{N}_{2}$ and the low-temperature phase of $o-\mathrm{H}_{2}, U_{0}>0$ and $U_{1}=0$ (more precisely, the second-degree term is zero, but higher-degree terms do exist); for $\beta-\mathrm{O}_{2}$, $U_{0}<0$ and $U_{1}>\left|U_{0}\right|$ and the order parameter is positive. For $\gamma-\mathrm{O}_{2}$, both molecular and crystal field constants are negative, a negative order parameter describes precession of disc-like molecules. The states with the negative order parameter can be treated as the orientational analog of the easyplane-type ordering in magnets.

As shown in Ref. 28, even very small crystal fields can substantially change the behavior of the system. For positive $U_{1}$, the main difference with the case of $U_{1}=0$ lies in the fact that the orientational phase transitions, instead of separating orientationally ordered and disordered states having generally speaking different symmetry, separate more and less ordered states of the same symmetry. Thus, these phase transitions are of the order-order type.

The most characteristic feature of the system at negative values of the crystal field (compared with that at $U_{1}=0$ ) is that states with a negative order parameter can exist as thermodynamically stable states of the system along with the states having a positive order parameter. The phase transitions occurring in the system at negative crystal fields are the transitions between two different ordered states, the easy-axis orientational states with the positive order parameter and the easy-plane orientational states with $\eta<0$.

In principle, the crystal-field term can be deduced from Eq. (3). It is proportional to a product of such quantities as $\xi_{c}=c / a-\sqrt{8 / 3}$, the deviation of $c / a$ from the ideal hcp value; $\xi_{m}=$ $=b / a-\sqrt{3}$, the deviation of $b / a$ from the ideal hcp value in the case of the monoclinic distortion, and $P_{2}\left(\cos \theta_{0}\right)=3 / 2 \cos ^{2} \theta_{0}-1 / 2$, where $\theta_{0}$ is the polar angle of the central molecule respective the $c$-axis. None of these quantities are known to sufficient accuracy either from experiment or theory. That is why the reduced crystal field will be treated in the present study as a parameter of theory.

\section{Results and discussion}

To find the phase diagram of the system of rotors described by the Hamiltonian [Eq. (1)], we used the same computational scheme as in Refs. 10,11. First, the energy spectrum of the linear rotors in the 
field $V Y_{20}$ was calculated, where $V=$ $=-\left(U_{0} \eta+U_{1}\right)$. We used the basis of spherical functions $Y_{l m}$ in which the kinetic energy operator $L^{2}$ is diagonal. The basis set was restricted to $l=7$, which, within the studied range of $V$, ensures sufficient accuracy of calculations for lower levels of the system. Making use of the spectrum obtained, we have calculated the free energy $F$ as a function of the order parameter and of the temperature at given values of the molecular field and crystal field constants $U_{0}$ and $U_{1}$. Then we found the temperature dependence of the order parameter from the condition that the free energy of the system is a minimum $(d F / d \eta=0)$. Data on the variation of the order parameter with temperature and molecular field constant $U_{0}$ allow to receive the phase transition line for the given value of the crystal field constant in the coordinates $U_{0}-T$. The locus of equation $d \eta / d T=\infty$ for different $U_{0}$ is the curve of absolute instability of the orientationally ordered phase. The locus of equation $F\left(\eta_{\mathrm{tr}}\right)=F(\eta=0)$ for different $U_{0}$ is the curve of the thermodynamically equilibrium transitions $\left(\eta_{\mathrm{tr}}\right.$ is the value of the order parameter for which the free energy of the ordered phase becomes equal to the free energy of the disordered phase).

Using van Kranendonk's analytical representation $[1,2]$ of the short-range valence potential of Ree and Bender [29], we have calculated the molecular field constant $U_{0}$ as a function of relative compression $R_{0} / R$, where $R_{0}$ and $R$ are the nearest neighbor distances at zero pressure and pressure $P$, respectively. It was assumed that the BS phase in solid HD has the same supposedly lowest energy structure $P c a 2_{1}$ as was predicted for solid $\mathrm{H}_{2}$ [2226]. Finally, to map the BSP transition line into $P-T$ space, we must use the pressure-volume equation of state. Although no $P-V$ experimental data have been reported for solid HD at these pressures, isotopic differences (at least between $\mathrm{H}_{2}$ and $\mathrm{D}_{2}$ ) in the megabar range are very small. Thus, we use the recent $\mathrm{x}$-ray pressure-volume equation of state for $\mathrm{H}_{2}$ and $\mathrm{D}_{2}$ measured by Loubeyre et al. [21] (Vinet functional form).

As expected, the phase transition pressure calculated under the assumption of zero crystal field underestimates the experimental value by a factor of four. As shown in our study of the model system given by the Hamiltonian [(Eq. (1)] [28], negative values for the crystal field shift the phase transition line upward to higher pressures. Figure 1, $a$, shows a set of theoretical curves that give the best agreement with the experimental data from Ref. 9. These curves correspond to reduced crystal fields $U_{1} / U_{0}$
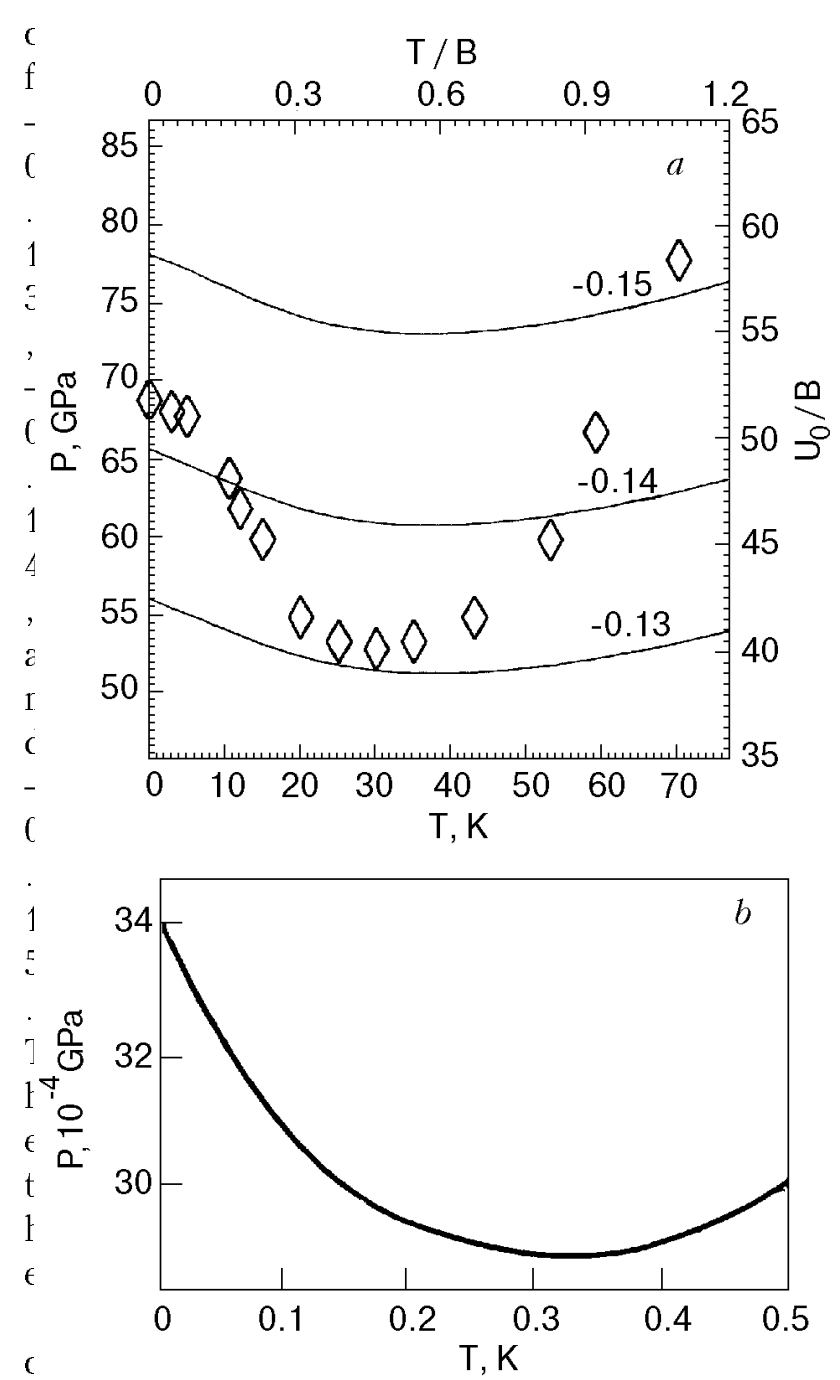

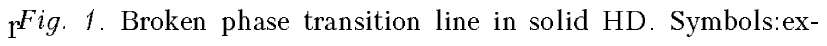
perimental data by Moshary, Chen, and Silvera [9]. Solid curves: present theory. Different curves are labelled by values of the reduced crystal field $(a)$. Melting curve in ${ }^{3} \mathrm{He}(b)$. u

c

ceeds in reproducing the distinguishing feature of the HD phase transition line, i. e. , the nonmonotonicity of the curve and a correct position of the minimum. The steeper temperature dependence characteristic of the experimental data is principally due to the effect of orientational correlations, and in part to the Heisenberg-like term in the HD-HD intermolecular potential omitted in this study.

In the present paper we consider the crystal field as a parameter in the theory, and thus the question exists as to the value and the sign of this parameter required to gain agreement between theory and experiment. As follows from the analysis of Eq. (3), the negative sign of the crystal field is definite, and the values given above correspond to the value of $\xi_{c}=c / a-\sqrt{8 / 3}$, the deviation of $c / a$ from the ideal hcp value, which was found in the recent $\mathrm{x}$-ray high-pressure study [21]. A detailed analysis 


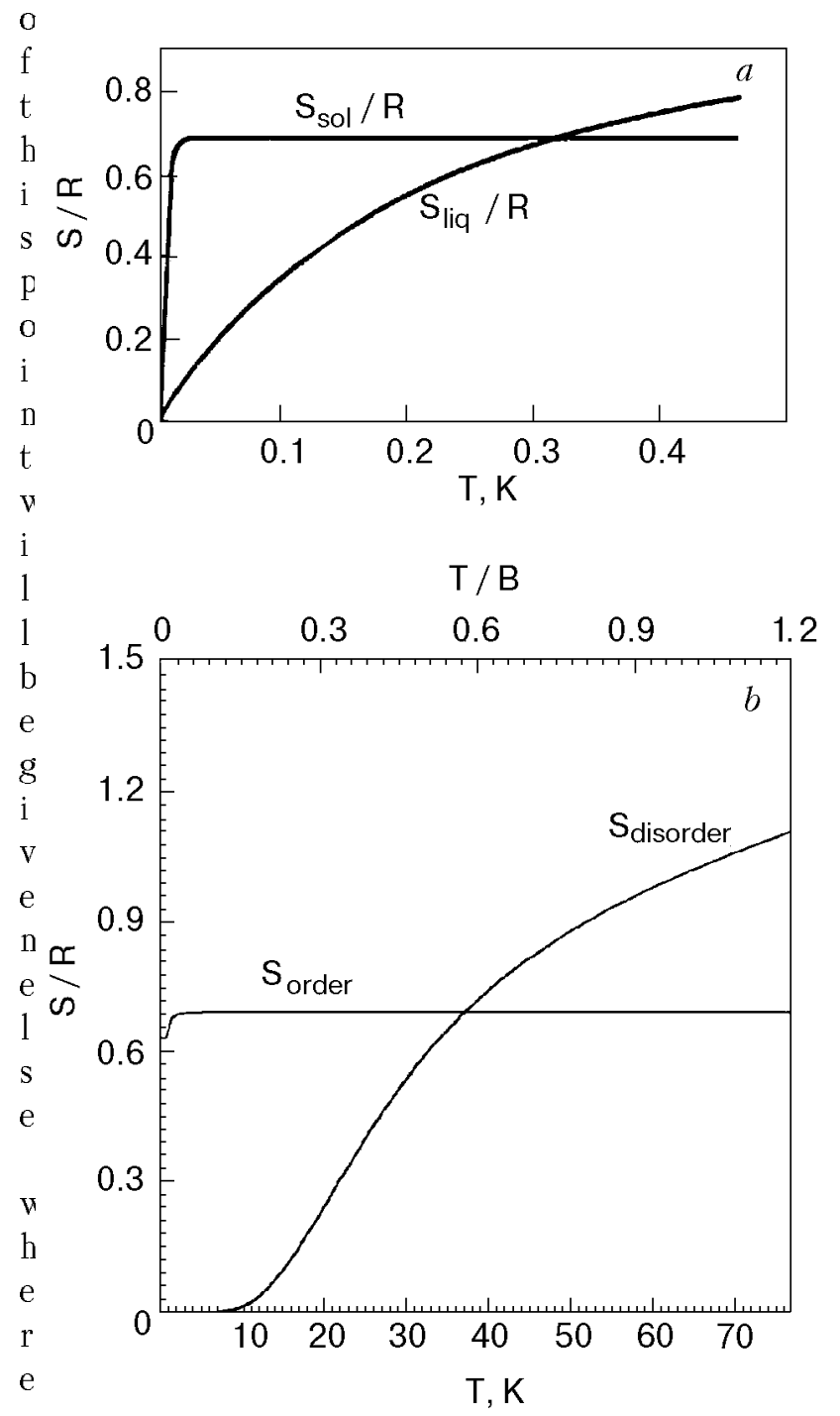

Hig. 2. Entropies of liquid and solid ${ }^{3} \mathrm{He}(a)$ and ordered and $\mathrm{e}^{\text {disordered }}$ phases of solid HD (b)

$\mathrm{r}$

e we would like to point out that the deformation of the lattice that gives rise to the negative crystal field as a response of the lattice to the applied pressure is in accord with the general Le-ChatelierBraun principle.

As one can see, the BSP transition line in solid HD is similar to the $P$ - $T$ melting curve of ${ }^{3} \mathrm{He}$ (Fig. 1,b). It is established that the presence of the minimum in the ${ }^{3} \mathrm{He}$ melting curve stems from the fact that the entropy of the solid phase exceeds the entropy of the liquid at low temperatures, where the thermal properties of the condensed phases are dominated by spin properties. Liquid ${ }^{3} \mathrm{He}$ obeys Fermi statistics, with the entropy proportional to the temperature. On the other hand, the entropy of solid ${ }^{3} \mathrm{He}$ is that of weakly interacting spin $1 / 2$ nuclei; that is, the entropy of solid ${ }^{3} \mathrm{He}$ is independent of temperature and equals to $R \ln 2$ (Fig.
$2, a)$. In this temperature region, the entropy contribution to the free energy is an additional factor that stabilizes the solid phase.

A similar situation occurs in the case of the system of rotors. The molecular field gives a doublet-shape ground state of the system; this provides an extra contribution to the entropy of the ordered phase, which is equal to $R \ln 2$. As a result, in the low-temperature region the entropy of the orientationally ordered phase is larger than that of the disordered phase (Fig. 2,b). Similar to the case of ${ }^{3} \mathrm{He}$, this is an additional factor that stabilizes the ordered phase. Above the point of intersection, the situation becomes «normal» and the entropy factor stabilizes the disordered phase.

\section{Conclusions}

The broken symmetry phase transition line in solid HD has been shown to be an example of quantum orientational melting. The unusual behavior of the phase transition line (i.e., its $P-T$ minimum) is a consequence of the symmetry properties of the system, namely, the fact that in HD all rotational states and transitions between them are allowed, in contrast to homonuclear isotopes $\mathrm{H}_{2}$ and $\mathrm{D}_{2}$. The systematic underestimation of the transition pressure characteristic of all theories of the BSP transition can be removed if crystal-field effects are taken into account. It was suggested that the effect of orientational correlations, and specifically for HD, the Heisenberg-like term in the intermolecular potential, should be taken into account to obtain quantitative agreement between theory and experiment.

\section{Acknowledgments}

This research was supported by the NSF (DMR9624050). The authors dedicate this work to Prof. $\mathrm{Yu}$. Kagan on the occasion of his 70th birthday.

1. V. G. Manzhelii and M. A. Strzhemechny, in: Physics of Cryocrystals, V. G. Manzhelii and Yu. A. Freiman (eds.), AIP Press, Woodbury, New York (1997).

2. J. van Kranendonk, Solid Hydrogen, Plenum Press, New York and London (1982).

3. J. Bigeleisen, J. Chem. Phys. 39, 769 (1963).

4. A. I. Prokhvatilov, M. A. Strzhemechny, and G. N. Shcherbakov, Low Temp. Phys. 19, 445 (1993).

5. M. A. Strzhemechny, Low Temp. Phys. 22, 92 (1996).

6. J. C. Raich and R. D. Etters, J. Low Temp. Phys. 6, 229 (1972).

7. I. F. Silvera and R. J. Wijngaarden, Phys. Rev. Lett. 47, 39 (1981).

8. H. E. Lorenzana, I. F. Silvera, and K. A. Goettel, Phys. Rev. Lett. 64, 1939 (1990). 
9. F. Moshary, N. H. Chen, and I. F. Silvera, Phys. Rev. Lett. 71, 3814 (1993).

10. Yu. A. Freiman, V. V. Sumarokov, A. P. Brodyanskii, and A. Jezowski, J. Phys.: Condens. Matter. 3, 3855 (1991).

11. A. P. Brodyanskii, V. V. Sumarokov, Yu. A. Freiman, and A. Jezowski, Low Temp. Phys. 19, 368 (1993).

12. Ho-kwang Mao and R. J. Hemley, Rev. Mod. Phys. 66, 671 (1994).

13. R. J. Hemley, A. F. Goncharov, H. K. Mao, E. Karmon, and J. H. Eggert, J. Low Temp. Phys. 110, 75 (1998).

14. N. H. Chen, E. Sterer, and I. F. Silvera, Phys. Rev. Lett. 76, 1663 (1996).

15. W. England, J. C. Raich, and R. D. Etters, J. Low. Temp. Phys. 22, 213 (1976).

16. J. Felsteiner and Z. Friedman, Phys. Rev. B8, 3996 (1973).

17. I. Aviram, S. Goshen, and R. Thieberger, J. Low Temp. Phys. 38, 371 (1980); ibid. 43, 549 (1981); ibid. 52, 397 (1983); ibid. 55, 349 (1984); Phys. Lett. 88 A, 410 (1982); J. Chem. Phys. 80, 5337 (1984).

18. Ad Lagendijk and I. F. Silvera, Phys. Lett. 84 A, 28 (1981).
19. M. Sprik and M. L. Klein, J. Chem. Phys. 81, 6207 (1984).

20. W. B. J. M. Janssen and A. van der Avoird, Phys. Rev. B42, 838 (1990)

21. P. Loubeyre, R. LeToullec, D. Hausermann, M. Hanfland, R. J. Hemley, H. K. Mao, and L. W. Finger, Nature 383, 702 (1996).

22. H. Nagara and T. Nakamura, Phys. Rev. Lett. 68, 2468 (1992).

23. H. Chacham, L. M. Falikov, and B. Koiller, Phys. Rev. B50, 7195 (1994).

24. K. Nagao, H. Nagara, and S. Matsubara, Phys. Rev. B56, 2295 (1997).

25. M. P. Surh, K. J. Runge, T. W. Barbee III, E. L. Pollock, and C. Mailhiot, Phys. Rev. B55, 11330 (1997).

26. J. Kohanoff, S. Scandolo, G. L. Chiarotti, and E. Tosatti, Phys. Rev. Lett. 78, 2783 (1997).

27. A. F. Goncharov, R. J. Hemley, H. K. Mao, and J. Shu, Phys. Rev. Lett. 80, 101 (1998).

28. Yu. A. Freiman, S. Tretyak, and A. Jezowski, J. Low Temp. Phys. 110, 147 (1998).

29. F. H. Ree and C. F. Bender, J. Chem. Phys. 71, 5362 (1979). 